\title{
On Two Families of Implicit Interval Methods of Adams-Moulton Type
}

\author{
Małgorzata Jankowska ${ }^{1}$ and Andrzej Marciniak ${ }^{2,3}$ \\ ${ }^{1}$ Poznań University of Technology, Institute of Applied Mechanics \\ Piotrowo 3, 60-965 Poznań, Poland \\ ${ }^{2}$ Poznań University of Technology, Institute of Computing Science \\ Piotrowo 3a, 60-965 Poznań, Poland \\ ${ }^{3}$ Adam Mickiewicz University, Faculty of Mathematics and Computer Science \\ Umultowska 87, 61-614 Poznań, Poland \\ e-mail:\{mjank/anmar\}@sol.put.poznan.pl
}

(Rec. 7 September 2005)

Abstract: In our previous paper [1] we have presented implicit interval methods of Adams-Moulton type. It appears that two families of these types of methods exist. We compare both families of methods and present a numerical example.

Key words: initial value problem, interval methods, floating-point interval arithmetic

\section{INTRODUCTION}

In 1980s the explicit interval methods of Adams-Bashforth type has been first introduced by Šokin, Kalmykov and Juldašev (see e.g. [5, 10]). The research on the multistep interval methods has been continued by Marciniak and Jankowska $[1-3,7,8]$. The implicit interval methods of Adams-Moulton type have been presented in [1]. In this paper we continue our previous considerations and specify a precise form of two different families of implicit interval methods of this kind. This distinction follows from some interval arithmetic properties. It also depends on which of two conventionally equivalent formulas is chosen as the basis of its interval counterpart (see Sec. 2). In Sec. 3 we consider the equation of motion of simple pendulum and present some numerical results. A short summary given in Sec. 4 brings this paper to the end.

\section{TWO FAMILIES OF IMPLICIT INTERVAL METHODS OF ADAMS-MOULTON TYPE} form

Let us consider the initial value problem (IVP) of the

$$
\begin{aligned}
& y^{\prime}=f(t, y), \quad 0 \leq t \leq \xi, \\
& y(0)=y_{0},
\end{aligned}
$$

where

$$
\xi \in \mathbf{R}, y=y(t) \in \mathbf{R}^{N}, \quad f:[0, \xi] \times \mathbf{R}^{N} \rightarrow \mathbf{R}^{N} .
$$

Let us choose a positive integer $m$ and select the mesh points $t_{i}=i h, i=0,1, \ldots, m$, where $h=\xi / m$. It is well-known (see e.g. [6]) that there are two equivalent formulas that describe the exact solution of (1) at $t_{n}, n=k, k+1, \ldots$, $m$. On the one hand we have

$$
\begin{gathered}
y\left(t_{n}\right)=y\left(t_{n-1}\right)+ \\
+h \sum_{j=0}^{k} \bar{\gamma}_{j} \nabla^{j} f\left(t_{n}, y\left(t_{n}\right)\right)+h^{k+2} \bar{\gamma}_{k+1} \bar{\psi}(\eta, y(\eta)),
\end{gathered}
$$

where

$$
\begin{gathered}
\nabla^{j} f\left(t_{n}, y\left(t_{n}\right)\right)= \\
=\sum_{m=0}^{j}(-1)^{m}\left(\begin{array}{c}
j \\
m
\end{array}\right) f\left(t_{n-m}, y\left(t_{n-m}\right)\right), \\
\bar{\gamma}_{0}=1, \quad \bar{\gamma}_{j}=\frac{1}{j !} \int_{-1}^{0} s(s+1) \ldots(s+j-1) d s, \\
j=1,2, \ldots, k+1,
\end{gathered}
$$


and

$$
\begin{gathered}
\bar{\psi}(\eta, y(\eta)) \equiv f^{(k+1)}(\eta, y(\eta)) \equiv y^{(k+2)}(\eta), \\
\eta \in\left[t_{n-k}, t_{n}\right] .
\end{gathered}
$$

On the other hand, since

$$
\begin{gathered}
\bar{\beta}_{k j}=(-1)^{j} \sum_{m=j}^{k}\left(\begin{array}{c}
m \\
j
\end{array}\right) \bar{\gamma}_{m}, \\
j=0,1, \ldots k,
\end{gathered}
$$

we can write (2) in the form

$$
\begin{gathered}
y\left(t_{n}\right)=y\left(t_{n+1}\right)+ \\
+h \sum_{j=0}^{k} \bar{\beta}_{k j} f\left(t_{n-j}, y\left(t_{n-j}\right)\right)+h^{k+2} \bar{\gamma}_{k+1} \bar{\psi}(\eta, y(\eta)) .
\end{gathered}
$$

Let us denote

$\Delta_{t}, \Delta_{y}$ - sets in which the function $f(t, y)$ is defined, i.e.

$$
\Delta_{t}=\{t \in \mathbf{R}: 0 \leq t \leq \xi\},
$$

$$
\begin{gathered}
\Delta_{y}=\left\{y=\left(y_{1}, y_{2}, \ldots, y_{N}\right)^{T} \in \mathbf{R}^{N}: \underline{b}_{i} \leq y_{i} \leq \bar{b}_{i}, \underline{b}_{i}, \bar{b}_{i} \in \mathbf{R},\right. \\
i=1,2, \ldots, N\},
\end{gathered}
$$

$F(T, Y)$ - interval extension of $f(t, y)$ (for the definition of interval extension see $e . g$. [4, 9-10]),

$\bar{\Psi}(T, Y)$ - interval extension of $\bar{\psi}(t, y)$ (see (5)).

Other assumptions about $F(T, Y)$ and $\bar{\Psi}(T, Y)$ are the same as in [1]. Furthermore, we denote by $I($.$) the space of$ intervals over (.).

Now, let us assume that $y(0) \in Y_{0}$ and the intervals $Y_{i}$ such that $y\left(t_{i}\right) \in Y_{i}, i=1,2, \ldots, k-1$, are known. Then, the implicit interval methods of Adams-Moulton type can be defined as follows (see also [1]):

$$
\begin{gathered}
Y_{n}=Y_{n-1}+h \sum_{j=0}^{k} \bar{\gamma}_{j} \nabla^{j} F_{n}+ \\
+h^{k+2} \bar{\gamma}_{k+1} \bar{\Psi}\left(T_{n}+[-k h, 0], Y_{n}+[-k h, 0] F\left(\Delta_{t}, \Delta_{y}\right)\right),(8) \\
n=k, k+1, \ldots, m,
\end{gathered}
$$

where $h=\xi / m, t_{i}=i h \in T_{i}, i=0,1, \ldots, m, \bar{\gamma}_{j}, j=0,1, \ldots, k+1$, are given by (4), $F_{n}=F\left(T_{n}, Y_{n}\right)$ and

$$
\nabla^{j} F_{n}=\sum_{m=0}^{j}(-1)^{m}\left(\begin{array}{c}
j \\
m
\end{array}\right) F_{n-m} .
$$

Moreover, the Eq. (8) can be written in the equivalent form as follows:

$$
\begin{gathered}
Y_{n}=Y_{n-1}+h \sum_{j=0}^{k} \bar{\gamma}_{j} F_{n}+h \sum_{j=1}^{k} \bar{\gamma}_{j} \sum_{m=1}^{j}(-1)^{m}\left(\begin{array}{c}
j \\
m
\end{array}\right) F_{n-m}+ \\
+h^{k+2} \bar{\gamma}_{k+1} \bar{\Psi}\left(T_{n}+[-k h, 0], Y_{n}+[-k h, 0] F\left(\Delta_{t}, \Delta_{y}\right)\right),(9) \\
n=k, k+1, \ldots, m .
\end{gathered}
$$

In particular for a given $k$ from (8) (or (9)) we get the following methods:

- $k=1$

$$
\begin{gathered}
Y_{n}=Y_{n-1}+\frac{h}{2}\left(2 F_{n}-F_{n}+F_{n-1}\right)+ \\
-\frac{h^{3}}{12} \Psi\left(T_{n}+[-h, 0], Y_{n}+[-h, 0] F\left(\Delta_{t}, \Delta_{y}\right)\right),
\end{gathered}
$$

- $k=2$

$$
\begin{gathered}
Y_{n}=Y_{n-1}+\frac{h}{12}\left(12 F_{n}-7 F_{n}+8 F_{n-1}-F_{n-2}\right)+ \\
-\frac{h^{4}}{24} \bar{\Psi}\left(T_{n}+[-2 h, 0], Y_{n}+[-2 h, 0] F\left(\Delta_{t}, \Delta_{y}\right)\right),
\end{gathered}
$$

- $k=3$

$$
\begin{gathered}
Y_{n}=Y_{n-1}+\frac{h}{24}\left(24 F_{n}-15 F_{n}+19 F_{n-1}-5 F_{n-2}+F_{n-3}\right)+ \\
-\frac{19}{720} h^{5} \bar{\Psi}\left(T_{n}+[-3 h, 0], Y_{n}+[-3 h, 0] F\left(\Delta_{t}, \Delta_{y}\right)\right) .
\end{gathered}
$$

Let us notice that for interval arithmetic the distributive law is not generally satisfied. However, since an interval is also a set, then for $X, Y, Z \in I(\mathbf{R})$, the following relation, as the subdistributive law, holds

$$
X \cdot(Y+Z) \subset X \cdot Y+X \cdot Z .
$$

Hence, the values of the interval extensions of $f$ in the above formulas with the same indices cannot be subtracted.

In real arithmetic we have

$$
\sum_{j=0}^{k} \bar{\beta}_{k j} f_{n-j}=\sum_{j=0}^{k} \bar{\gamma}_{j} \nabla^{j} f_{n},
$$

where $f_{n-j}=f\left(t_{n-j}, y\left(t_{n-j}\right)\right), \quad j=0,1, \ldots, k$. Hence, the formula (2) is equivalent to (7). But in interval arithmetic we have

$$
\sum_{j=0}^{k} \bar{\beta}_{k j} F_{n-j} \subset \sum_{j=0}^{k} \bar{\gamma}_{j} \nabla^{j} F_{n}
$$

where the subset relation $(\subset)$ is defined as not necessarily proper, and we get another kind of implicit interval methods corresponding to the conventional formula (7), namely 


$$
\begin{gathered}
Y_{n}=Y_{n-1}+h \bar{\beta}_{k 0} F_{n}+h \sum_{j=1}^{k} \bar{\beta}_{k j} F_{n-j}+ \\
+h^{k+2} \bar{\gamma}_{k+1} \bar{\Psi}\left(T_{n}+[-k h, 0], Y_{n}+[-k h, 0] F\left(\Delta_{t}, \Delta_{y}\right)\right), \\
n=k, k+1, \ldots, m,
\end{gathered}
$$

where $h=\xi / m, t_{i}=i h \in T_{i}, i=0,1, \ldots, m$, and $\bar{\beta}_{k j}, j=0,1, \ldots, k$ are given by (6). In particular for a given $k$ from (12) we get the following methods:

- $k=1$

$$
\begin{gathered}
Y_{n}=Y_{n-1}+\frac{h}{2}\left(F_{n}+F_{n-1}\right)+ \\
-\frac{h^{3}}{12} \bar{\Psi}\left(T_{n}+[-h, 0], Y_{n}+[-h, 0] F\left(\Delta_{t}, \Delta_{y}\right)\right),
\end{gathered}
$$

- $k=2$

$$
\begin{gathered}
Y_{n}=Y_{n-1}+\frac{h}{12}\left(5 F_{n}+8 F_{n-1}-F_{n-2}\right)+ \\
-\frac{h^{4}}{24} \bar{\Psi}\left(T_{n}+[-2 h, 0], Y_{n}+[-2 h, 0] F\left(\Delta_{t}, \Delta_{y}\right)\right),
\end{gathered}
$$

- $k=3$

$$
\begin{gathered}
Y_{n}=Y_{n-1}+\frac{h}{24}\left(9 F_{n}+19 F_{n-1}-5 F_{n-2}+F_{n-3}\right)+ \\
-\frac{19}{720} h^{5} \bar{\Psi}\left(T_{n}+[-3 h, 0], Y_{n}+[-3 h, 0] F\left(\Delta_{t}, \Delta_{y}\right)\right) .
\end{gathered}
$$

If we denote by $Y_{n}^{1}$ the interval-solutions obtained from the formula (8) (or (9)), i.e. from the formula with backward interval differences, and by $Y_{n}^{2}$ the interval-solutions obtained from (12), then, we can prove

Theorem 1. $Y_{n}^{2} \subset Y_{n}^{1}$,

which means that the second kind of implicit interval formulas gives the interval-solution with a smaller width, i.e. it is better. The proof of the Theorem (1) follows immediately from (11).

Let us note that (8) (or (9)) and (12) are nonlinear interval equations with respect to $Y_{n}, n=k, k+1, \ldots, m$. It implies that in each step of implicit interval methods we have to solve an interval equation of the form

$$
Y=G(T, Y),
$$

where

$T \in I\left(\Delta_{t}\right) \subset I(\mathbf{R}), \quad Y=\left(Y_{1}, Y_{2}, \ldots, Y_{N}\right)^{T} \in I\left(\Delta_{y}\right) \subset I\left(\mathbf{R}^{N}\right)$,

$$
G: I\left(\Delta_{t}\right) \times I\left(\Delta_{y}\right) \rightarrow I\left(\mathbf{R}^{N}\right) .
$$

If we assume that the function $G$ is a contracting mapping, then the well-known fixed-point theorem implies that the iteration process

$$
Y^{(l+1)}=G\left(T, Y^{(l)}\right), \quad l=0,1, \ldots
$$

is convergent to $Y^{*}$, i.e. $\lim _{l \rightarrow \infty} Y^{(l)}=Y^{*}$, for an arbitrary choice of $Y^{(0)} \in I\left(\Delta_{y}\right)$.

For the interval methods of Adams-Moulton type given by (8) (or (9)), the iteration process (13) is of the form

$$
\begin{gathered}
Y_{n}^{(l+1)}=Y_{n-1}+h \sum_{j=0}^{k} \bar{\gamma}_{j} F\left(T_{n}, Y_{n}^{(l)}\right)+ \\
+h \sum_{j=1}^{k} \bar{\gamma}_{j} \sum_{m=1}^{j}(-1)^{m}\left(\begin{array}{c}
j \\
m
\end{array}\right) F\left(T_{n-m}, Y_{n-m}\right)+ \\
+h^{k+2} \bar{\gamma}_{k+1} \bar{\Psi}\left(T_{n}+[-k h, 0], Y_{n}^{(l)}+\right. \\
\left.+[-k h, 0] F\left(\Delta_{t}, \Delta_{y}\right)\right), l=0,1, \ldots, n=k, k+1, \ldots, m,
\end{gathered}
$$

and for the methods (12) of the form

$$
\begin{gathered}
Y_{n}^{(l+1)}=Y_{n-1}+h \bar{\beta}_{k 0} F\left(T_{n}, Y_{n}^{(l)}\right)+ \\
+h \sum_{j=1}^{k} \bar{\beta}_{k j} F\left(T_{n-j}, Y_{n-j}\right)+ \\
+h^{k+2} \bar{\gamma}_{k+1} \bar{\Psi}\left(T_{n}+[-k h, 0],\right. \\
\left.Y_{n}^{(l)}+[-k h, 0] F\left(\Delta_{t}, \Delta_{y}\right)\right), l=0,1, \ldots, n=k, k+1, \ldots, m .
\end{gathered}
$$

In (14) and (15) we usually choose $Y_{n}^{(0)}=Y_{n-1}$.

\section{NUMERICAL EXAMPLE}

Let us consider the motion of simple pendulum. The adequate equation is of the form

$$
\ddot{\varphi}+u^{2} \sin \varphi=0
$$

where $\varphi=\varphi(t), \quad u=\sqrt{g / L}, g$ is the gravitational acceleration at Earth's surface and $L$ denotes the pendulum length.

Denoting $y_{1}=\dot{\varphi}, \quad y_{2}=\varphi$, where $y_{1}=y_{1}(t), y_{2}=y_{2}(t)$, we transform (16) with the initial conditions $\dot{\varphi}(0)=0$, $\varphi(0)=\varphi_{0}$, into the following system of differential equations of the first order:

$$
\dot{y}_{1}=-u^{2} \sin y_{2}, \quad \dot{y}_{2}=y_{1},
$$

with the initial conditions

$$
y_{1}(0)=0, \quad y_{2}(0)=\varphi_{0} .
$$

We have integrated (17) with (18) for $t=1$ [s], where $\varphi_{0}=$ $\pi / 60$ [rad], $g=9.81\left[\mathrm{~m} / \mathrm{s}^{2}\right], L=1[\mathrm{~m}]$. We have used the interval methods of Adams-Moulton type (8) and (12) 
for the number of method steps $k=1,2,3$, and for the stepsizes $h=1 \mathrm{E}-3,1 \mathrm{E}-4,1 \mathrm{E}-5,1 \mathrm{E}-6$.

In Figures 1 and 2 the comparison of the widths of two considered families is given. The widths of interval-solutions $Y_{i}(1), i=1,2$, obtained with the IIAM methods (12) are always smaller than the ones that have been obtained by the IIAM methods (8) (or (9)) for the same

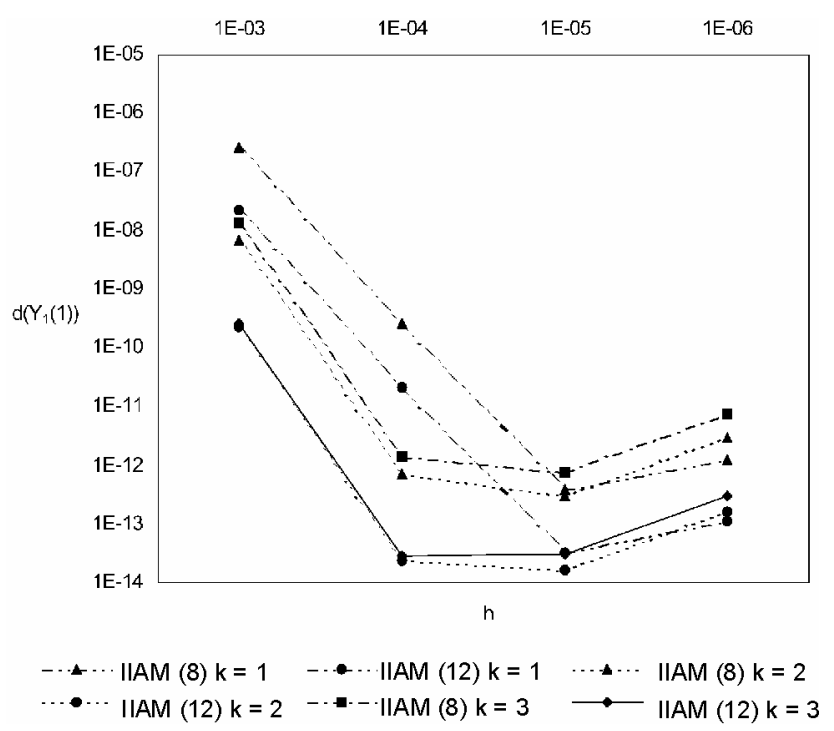

Fig. 1. Width of the interval-solution $Y_{1}(1)$ obtained by the IIAM methods (8) and (12) for (17) with (18), where $k=1,2,3$, vs. the stepsize $h$. IIAM - the implicit interval methods of Adams-Moulton type

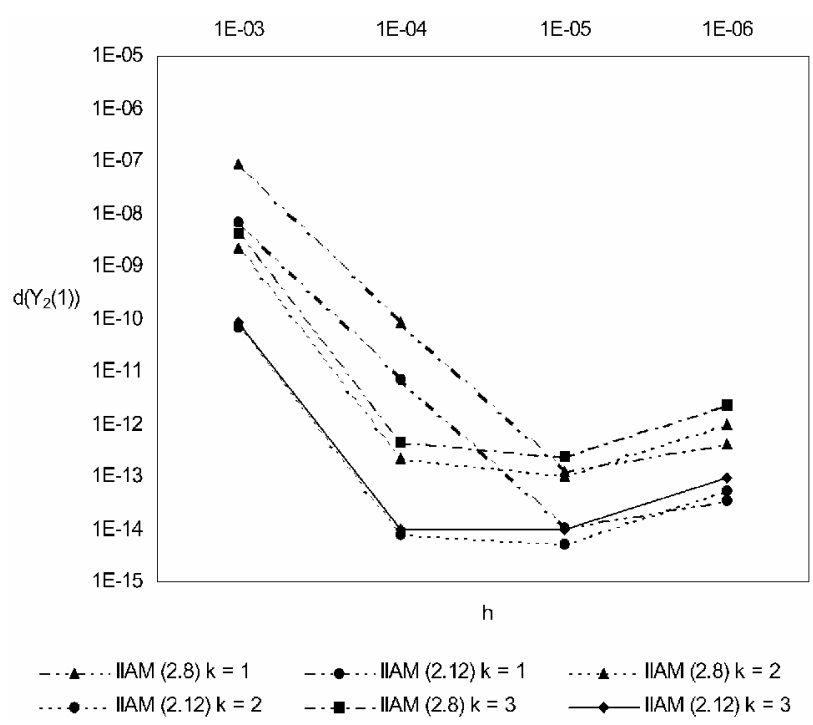

Fig. 2. Width of the interval-solution $Y_{2}(1)$ obtained by the IIAM methods (8) and (12) for (17) and (18), where $k=1,2,3$, vs. the stepsize $h$. IIAM - the implicit interval methods of Adams-Moulton type parameter $k$ and stepsize $h$. The increase of parameter $k$ for the same stepsize $h$ contributes to the improvement of interval-solutions, i.e. decrease in their widths. The similar effect can be achieved if we reduce the stepsize $h$ for the same value of parameter $k$. On the other side, let us notice that the widths of interval-solutions obtained by the IIAM methods (8) (or (9)) and (12) for $k=3$ are somewhat worse that the once that have been obtained by the considered methods for $k=2$. The similar effect can be observed for excessive decrease in the stepsize $h$. Hence, one can conclude that the optimal stepsize $h$ and number of method steps $k$ should be chosen for each particular interval method and each IVP.

\section{SUMMARY}

The main aim of our paper is to specify the precise form of two families of implicit interval methods of AdamsMoulton type. The reason for the existence of the second family has been explained in Sec. 2. The results of numerical experiment given is Sec. 3 have confirmed the thesis of Theorem 1. Furthermore, we have explained why the formulas (12) rather then (8) (or (9)) should be used in interval computations.

\section{References}

[1] M. Jankowska and A. Marciniak, Implicit Interval Multistep Methods for Solving the Initial Value Problem, CMST 8(1), 17-30 (2002).

[2] M. Jankowska and A. Marciniak, On Explicit Interval Methods of Adams-Bashforth Type, CMST 8(2), 46-57 (2002).

[3] M. Jankowska and A. Marciniak, Preliminaries of the IMM System for Solving the Initial Value Problem by Interval Multistep Methods [in Polish], Pro Dialog 10, 117-134 (2005).

[4] L. Jaulin, M. Kieffer, O. Didrit and É. Walter, Applied Interval Analysis, Springer-Verlag, London 2001.

[5] S. A. Kalmykov, Ju. I. Šokin and E. Ch. Juldašev, Methods of Interval Analysis [in Russian], Nauka, Novosibirsk 1986.

[6] A. Krupowicz, Numerical Methods of Initial Value Problems of Ordinary Differential Equations [in Polish], PWN, Warsaw 1986.

[7] A. Marciniak, Implicit Interval Methods for Solving the Initial Value Problem, Numerical Algorithms 37, 241-251 (2004).

[8] A. Marciniak, On Multistep Interval Methods for Solving the Initial Value Problem, Journal of Computational and Applied Mathematics (in press).

[9] R. E. Moore, Interval Analysis, Prentice-Hall, Englewood Cliffs 1966.

[10] Ju. I. Šokin, Interval Analysis [in Russian], Nauka, Novosibirsk 1981. 


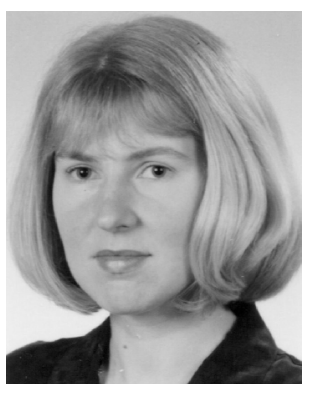

Malgorzata Jankowska received the Ph.D. degree in Computer Science (specialization of Numerical Methods) from the Poznań University of Technology (Poland) in 2006. Since 2003 she works in Institute of Applied Mechanics, Poznań University of Technology. She is a member of Polish Association of Theoretical and Applied Mechanics. Her current research concern the numerical aspects of scientific computing with emphasis on interval multistep methods for solving the IVP for ODEs and their implementation in the $\mathrm{C}++$ language.

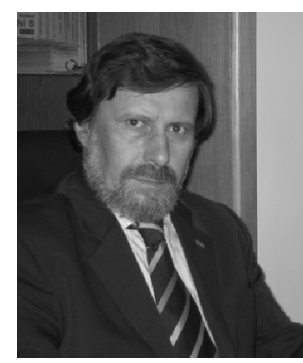

Andrzej MarciniaK, Associate Professor (Ph. D. 1981, Dr. habil. 1993), in years 1977-1986 in the Institute of Mathematics, Adam Mickiewicz University of Poznan, in years 1986-1992 in the Institute of Mathematics, Poznan University of Technology, since 1992 in the Institute of Computing Science, and since 2000 also in the Faculty of Mathematics and Computer Science, Adam Mickiewicz University of Poznan. In years 19821983 visiting professor in the University of Florida in Gainesville, and visiting professor in the University of Texas at Arlington in 1990. Specialist in computer programming and numerical analysis, especially in numerical methods for solving differential equations (including interval methods) and their application in celestial mechanics. Author or co-author of 3 monographs, 30 textbooks, and over 40 papers in professional journals and conference proceedings. Editor-in-Chief of the Microcomputer User's Library of NAKOM Publisher, and Pro Dialog journal - an official scientific journal of the Polish Information Processing Society. Vice-president (in 1999-2005) and President (since 2005) of the Polish Information Processing Society. Member of the State Acreditation Committee (since 2005). 